\title{
The ROSAT/NVSS AGN sample
}

\section{Gurgen M. Paronyan, Hayk V. Abrahamyan, Gohar S. Harutyunyan and Areg M. Mickaelian}

Byurakan Astrophysical Observatory (BAO), Byurakan 0213, Aragatzotn province, Armenia Email: gurgen@bao.sci.am, abrahamyanhayk@gmail.com, goharutyunyan@gmail.com, aregmick@aras .am

\begin{abstract}
We attempt to create an X-ray/radio AGN catalog and make its multiwavelength studies. ROSAT Bright Source Catalogue (BSC) contains 18,806 and ROSAT Faint Source Catalogue (FSC), 105,922 X-ray sources giving the total number of ROSAT X-ray sources 124,727 (one source is listed twice). On the other hand, NVSS radio catalogue contains 1,773,484 sources. Taking into account that X-ray sources contain AGN, bright stars and galaxies, clusters, white dwarfs (WD), cataclysmic variables (CV), etc., the cross-identification with radio catalogue may distinguish the extragalactic sources. We have cross-correlated ROSAT catalogs with NVSS one with a search radius 30 arcsec. 9,193 associations have been found. To distinguish AGN from the normal bright galaxies and clusters, Veron-Cetty \& Veron AGN catalog (v.13, 2010; VCV13) containing 168,940 objects have been used. A cross-correlation of the 9,193 ROSAT/NVSS sources with the VCV-13 with a search radius 30 arcsec resulted in 3,094 associations. Thus we are left with more 6,099 X-ray/radio sources without an optical identification. Brighter objects are normal bright galaxies, while we believe that all faint ones are candidate AGN with some contamination of distant clusters. SDSS spectroscopic survey allows us classify objects by activity types, and a number of our candidate AGN is found to be present in SDSS. We attempt to find connections between the fluxes in different wavelength ranges, which will allow us to confirm AGN and blazars candidates and in some cases find new ones.
\end{abstract}

Keywords. X-ray and radio surveys, SDSS, cross-correlations, AGN, AGN candidates

We attempt to create an X-ray/radio AGN catalog and make its multiwavelength (MW) studies in order to find connections between the fluxes in different wavelength ranges, which will allow us to confirm AGN candidates and in some cases find new ones. For this, ROSAT Bright Source (BSC, Voges et al. 1999) and Faint Source (FSC, Voges et al. 2000) catalogues for X-ray, and NVSS catalogue (Condon et al. 1998) for radio sources have been used. ROSAT BSC contains 18,806 and ROSAT FSC contains 105,922 $\mathrm{X}$-ray sources. However, as one source is listed twice, the total number of ROSAT X-ray sources is 124,727 . On the other hand, NVSS contains 1,773,484 sources. Taking into account that X-ray sources contain AGN, bright stars and galaxies, clusters, white dwarfs (WD), cataclysmic variables (CV) etc., the cross-identification with radio catalogue may distinguish the extragalactic sources, excluding all stars.

We have cross-correlated both ROSAT catalogs with NVSS with a typical search radius 30 arcsec. However, a new cross-correlation software was used allowing matching objects by their individual positional errors and taking associations within $3 \sigma .9,193$ ROSAT/NVSS associations have been found. To distinguish AGN from normal bright galaxies and clusters, Veron-Cetty \& Veron AGN catalog (Veron-Cetty \& Veron 2010) containing 168,940 objects was used. The cross-correlation of 9193 ROSAT/NVSS sources with the VCV-13 with a search radius 30 arcsec resulted in 3,094 associations, including QSOs, blazars, all types of Seyfert galaxies, LINERs and HII regions. As a result, we were left with more 6,099 X-ray/radio sources without an optical identification. 
Brighter objects are normal bright galaxies having been detected in both ROSAT and NVSS, while we believe that all faint ones are candidate AGN with some contamination of distant clusters (this assumption is based on optical identifications of ROSAT sources by means of Hamburg Quasar Survey (HQS) low-dispersion spectra; Zickgraf et al. 2003; Mickaelian et al. 2006). SDSS DR10 spectroscopic survey (Ahn et al. 2013) allows us classify objects by activity types, and a number of our candidate AGN is found to be present in SDSS.

For all 9,193 identified sources we have made multiwavelength investigations in several wavelength ranges (gamma-ray, X-ray, UV, optical, IR, radio) using FERMI (Nolan et al. 2012), INTEGRAL (Bird et al. 2010), GALEX (Bianchi et al. 2011), APM (McMahon et al. 2000), SDSS, 2MASS (Cutri et al. 2003), WISE (Wright et al. 2010; Cutri et al. 2012), IRAS (Moshir et al. 1992) and other catalogs. We calculated the absolute magnitudes, fluxes, improved the coordinates and redshifts.

An attempt is made to find a connection between the radiation fluxes in different wavelength bands for different types of sources and identify their characteristics, thus confirming all candidate AGN. MW SEDs have been built for these sources using 25 photometric points from gamma-ray to radio, most of them having at least 15-20 measurements.

Candidate blazars sample has been compiled based on Rome Blazars Catalogue (BZCAT, Massaro et al. 2009) and adding many new candidate blazars, as presence of both $\mathrm{X}$-ray and radio indicate high probability of being a blazar. For these sources, we have carried out a study for their optical and radio variability based on a comparison of their USNO-B1.0 (Monet et al. 2003) POSS1 and POSS2 epochs magnitudes for optical range and NVSS and FIRST fluxes for radio range. The photometric accuracy for each object individually has been estimated and those having flux differencies larger than $3 \sigma$ have been considered as variables. Spectrosocpic observations should be carried out to confirm these objects as blazars and in this case the total number of all blazars may be doubled.

\section{References}

Ahn, C. P., Alexandroff, R., Allende Prieto, C., et al. 2013, ApJS, in press.

Bianchi L., Herald J., Efremova B., et al. 2011 ApSS 335, 161

Bird A. J., Bazzano A., Bassani L., et al. 2010 ApJSS 186, 1

Condon J. J., Cotton W. D., Greisen E. W., et al. 1998, Astron. J., 115, 1693

Cutri, R. M., Skrutskie, M. F., van Dyk, S., et al. 2003 IPAC/CalTech

Cutri R. M., Wright E. L., Conrow T., et al. 2012, WISE All-Sky DR, VizieR Catalog II/311

Massaro E., Giommi P., Leto C., et al. 2009, A\&A 495, 691

McMahon, R. G., Irwin, M. J., \& Maddox, S. J., 2000, IoA, Cambridge, UK

Mickaelian A. M., Hovhannisyan L. R., Engels D., Hagen H.-J., \& Voges W. 2006, A $\& A 449$, 425

Monet D. G., Levine S. E., Casian B., et al. 2003, AJ 125, 984

Moshir M., Kopan G., Conrow T., et al. 1992, ES IRAS FSS, v2, JPL

Nolan P. L., Abdo A. A., Ackermann M., et al. 2012 ApJSS 199, 31N

Veron-Cetty M. P. \& Veron P. 2010, Astron. Astrophys., 518, A10

Voges W., Aschenbach B., Boller T., et al. 1999, Astron. Astrophys, 349, 389

Voges W., Aschenbach B., Boller Th., et al. 2000, IAU Circ, 7432

\&Wright, E. L., Eisenhardt, P. R. M., \& Mainzer, A. K., 2010, AJ 140, 1868

Zickgraf F.-J., Engels D., Hagen H.-J., Reimers D., \& Voges W. 2003, A\&A 406, 535 